\section{Fuzzy logic}

B. Gurtner

Die ersten Fotoapparate mit Autofokus hatten ihre Tücken. Stand im Hintergrund scharf gezeichnet das Matterhorn und nahte im Gegenlicht ein zottiger Ziegenbock, so ging der Schnappschuss nicht rechtzeitig los. Die Automatik suchte für Berg und Bock eine perfekte Einstellung und zoomte zwischen Geiss und Gipfel solange hin und her, bis der beste Moment verpasst war. Die Fokussierung geriet vollends ausser Kontrolle, wenn ein Gittermuster vor die Linse kam, weil die Distanzmessung abwechselnd durch die Maschen fiel oder im Geflecht hängenblieb.

Kameras gehörten denn auch zu den ersten Anwendungsgebieten der "fuzzy logic», die mit künstlicher Intelligenz das abwägende menschliche Denken und Entscheiden nachzuahmen sucht. Ein praktikables Optimum ist nützlicher als das unerreichbare Maximum. Inzwischen werden Bahnen, Flugzeuge, Verkehrssignale, automatisierte Produktionsanlagen und auch medizinische Geräte mit Software gesteuert, die nicht einer haarscharfen kartesianischen Logik folgt, sondern kompromissfördernde mathematische Konzepte verwirklicht, wie sie unter anderen Professor Lotfi Zadeh vor gut 30 Jahren in Berkeley erdacht hat.

Wenn die Polizei einen Vermissten "von mittelgrosser Statur" meldet, ist dieses Signalement mindestens so hilfreich wie "172 cm gross", da niemand mit einem Messband auf die Suche gehen wird. Medizinische Diagnosenfahnder fordern oft um so präzisere Laborwerte, je diffuser ihre Vorstellungen sind und neigen dazu, Befunde an der Grenze des Normalbereichs stets im Sinne ihrer Hypothesen zu interpretieren: 4\% Eosinophile «beweisen» wunschgemäss eine Allergie oder sind «belanglos», wenn sie nicht ins diagnostische Konzept passen. Dabei wird kaum je beachtet, dass eine Nachzählung von 1000 statt nur 200 Leukozyten im gleichen Blutausstrich lediglich 2\% oder doch 6\% Eosinophile ergeben könnte.

Die wissenschaftliche Wahrheit unterliegt nicht erst seit Kopernikus und Galilei Halbwertzeiten und Verfalldaten. Das momentan gültige Weltbild hat immer ausgefranste Ränder. Für Computer hingegen gibt es nur 1 oder 0, Sein oder Nicht-Sein. Und es scheint, dass sich heute auch im ärztlichen Denken digitale Schaltungen anzubahnen beginnen. Wenn jedoch Aufmerksamkeit und Überlegungen frei krei-

Korrespondenz:

Dr. med. Bernhard Gurtner

Eggstrasse 76

CH-8620 Wetzikon

E-mail: gurtner.bernhard@bluewin.ch sen können wie eine Radarantenne, kommt das gesuchte Ziel eher ins Blickfeld, als wenn die Weichen einer algorithmischen Geleiseanlage starr und definitiv immer nur in eine von zwei Richtungen gestellt werden dürfen. An jeder Verzweigung des Entscheidungsbaumes hat man sich auf JA oder NEIN festzulegen, wo doch ein VIELLEICHT oder WEISS NICHT der Wirklichkeit angemessener wäre. So werden Diagnosen vorschnell und definitiv verworfen, obschon nicht nur EBM-Freunden bewusst ist, dass sehr wenige Untersuchungen eine 100\%ige Aussage erlauben. Dennoch werden Patienten "zum Ausschluss" eingewiesen und "nach Ausschluss» frühzeitig entlassen. Für englischsprachige Autoren sind Lungenembolien «ruled out», wobei sie sich im gleichen Paper über die ungenügende Sensitivity der Testmethoden beklagen. Die ausschliessende Haltung ist nicht nur anmassend, sondern lebensgefährlich, wenn sie dazu verleitet, begründete Verdachtsdiagnosen unwiderruflich abzuhaken. Nach angeblichem Ausschluss eines Hirntumors im CT, eines Herzinfarkts im EKG, eines Aneurysmas im Angiogramm sind uns Patienten an genau den Erkrankungen gestorben, die infolge apparativen oder menschlichen Unvermögens verpasst und apodiktisch als nicht existent bezeichnet worden waren.

Sagen und schreiben wir doch vorsichtiger, dass keine Anhaltspunkte für eine vermutete Diagnose gefunden werden konnten. Ein berechtigter Verdacht soll hartnäckig weiter verfolgt werden. Mit «fuzzy logic" wird kein voreiliger Ja-Nein-Schlussstrich gezogen, sondern der Wahrscheinlichkeit eine Türe offengelassen, «absence of evidence is not evidence of absence".

Viele Ärztinnen und Ärzte erleben den Wechsel von der Kliniktätigkeit in die Praxis als Wirklichkeitsschock. Im Spital wurde alles "abgeklärt" und "ausgeschlossen", ehe eine gezielte Therapie eingeleitet werden durfte. In der Praxis muss man sich oft mit einer ungesicherten Vermutung begnügen, und die Behandlung beginnen, noch bevor die Diagnose steht. Ex juvantibus zeigt sich dann bestenfalls, ob der erste intuitive Eindruck zutreffend war. Etwas gar fuzzy und von seltsamer Logik war allerdings die taktische Anweisung eines Landarztes an seinen noch unerfahrenen, schulmässig vorgehenden Praxisvertreter: "Bei Fieber nicht lange fragen oder untersuchen, sondern sofort Penicillin spritzen! Hilft es, war die Diagnose richtig, wenn nicht, gehört der Fall sowieso ins Kantonsspital!»

Eine Sepsis kann auch nach sechs negativen Blutkulturen und einer unauffälligen Echokardiographie nicht endgültig ausgeschlossen werden. Das wissen alle, doch behauptet ein geläufiger Slang immer wieder das Gegenteil. Wie wäre es mit einer kleinen Sprachreform? "Nach Ausschluss» ist eine leichtfertige Redewendung, die durch «keine Hinweise für» ersetzt werden müsste. Wer ohne Pilze aus dem Wald zurückkommt, kann niemals sicher sein, ob er im dunklen Forst nicht doch einige verwertbaren Schwämme übersehen hat. 